\title{
Retraction Note: The impact of repeated vaccination on influenza vaccine effectiveness: a systematic review and meta-analysis
}

\author{
Lauren C. Ramsay', Sarah A. Buchan², Robert G. Stirling ${ }^{2,3}$, Benjamin J. Cowling ${ }^{4}$, Shou Feng ${ }^{4}$, \\ Jeffrey C. Kwong ${ }^{1,2,5,6,7}$ and Bryna F. Warshawsky ${ }^{8,9^{*}}$
}

\section{Retraction Note}

The authors have retracted this article, The impact of repeated vaccination on influenza vaccine effectiveness: a systematic review and meta-analysis. Upon reviewing the published work, the authors determined that there were a few erroneous inclusions and an omission of estimates which impacted the conclusion for the influenza $A(H 3 N 2)$ and influenza $\mathrm{B}$ analyses comparing vaccination in both years to the current year only. All authors agree to this retraction, and they have been given the opportunity to submit a revised version in the journal.

\footnotetext{
Author details

'Public Health Ontario, 480 University Avenue Suite 300, Toronto, ON M5G 1V2, Canada. ${ }^{2}$ Dalla Lana School of Public Health, University of Toronto, 155 College St, Toronto, ON M5T 3M7, Canada. ${ }^{3}$ Public Health Agency of Canada, 130 Colonnade Road, Ottawa, ON K1A OK9, Canada. ${ }^{4}$ WHO Collaborating Centre for Infectious Disease Epidemiology and Control, School of Public Health, Li Ka Shing Faculty of Medicine, The University of Hong Kong, Pokfulam, Hong Kong, China. ${ }^{5}$ Institute for Clinical Evaluative Sciences, Veterans Hill Trail, 2075 Bayview Avenue G1 06, Toronto, ON M4N 3M5, Canada. ${ }^{6}$ Department of Family \& Community Medicine, University of Toronto, 155 College St, Toronto, ON M5T 3M7, Canada. ${ }^{7}$ University Health Network, 399 Bathurst St, Toronto, ON M5T 2S8, Canada. ${ }^{8}$ Public Health Ontario, 480 University Avenue Suite 300, Toronto, ON M5G 1V2, Canada. ${ }^{9}$ Department of Epidemiology and Biostatistics, Western University, 1151 Richmond St, London, ON N6A 3K7, Canada.
}

Received: 18 July 2018 Accepted: 25 July 2018

Published online: 12 August 2018

\section{Reference}

1. Ramsay $L C$, et al. The impact of repeated vaccination on influenza vaccine effectiveness: a systematic review and meta-analysis. BMC Med. 2017;15:159. https://doi.org/10.1186/s12916-017-0919-0

\footnotetext{
* Correspondence: bryna.warshawsky@oahpp.ca

${ }^{8}$ Public Health Ontario, 480 University Avenue Suite 300, Toronto, ON M5G 1V2, Canada

${ }^{9}$ Department of Epidemiology and Biostatistics, Western University, 1151 Richmond St, London, ON N6A 3K7, Canada
}

(c) The Author(s). 2018 Open Access This article is distributed under the terms of the Creative Commons Attribution 4.0 International License (http://creativecommons.org/licenses/by/4.0/), which permits unrestricted use, distribution, and reproduction in any medium, provided you give appropriate credit to the original author(s) and the source, provide a link to the Creative Commons license, and indicate if changes were made. The Creative Commons Public Domain Dedication waiver (http://creativecommons.org/publicdomain/zero/1.0/) applies to the data made available in this article, unless otherwise stated. 